\title{
A STUDY OF BACTERIAL ISOLATES AND THEIR SENSITIVITY PATTERN TO ANTIBIOTICS IN EMPYEMA THORACIS CASES IN A TERTIARY CARE HOSPITAL
}

\author{
Amiya Kumar Dwari¹, Sumanta Jha², Somnath Sarkar³, Swapnendu Misra4, Subhankar Chakraborty5, Abhijit Mandal ${ }^{6}$ \\ ${ }_{1}^{1}$ Associate Professor, Department of Respiratory Medicine, NRS Medical College, Kolkata, West Bengal, India. \\ ${ }^{2}$ Assistant Professor, Department of Respiratory Medicine, NRS Medical College, Kolkata, West Bengal, India. \\ 3Medical Officer, Swasthya Bhawan, Kolkata, West Bengal, India. \\ ${ }_{4}^{4}$ Assistant Professor, Department of Respiratory Medicine, NRS Medical College, Kolkata, West Bengal, India. \\ ${ }_{5}^{5}$ Medical Officer (Specialist), Department of Respiratory Medicine, NRS Medical College, Kolkata, West Bengal, India. \\ ${ }^{6}$ Professor, Department of Respiratory Medicine, NRS Medical College, Kolkata, West Bengal, India.
}

\begin{abstract}
BACKGROUND
ABSTRACT

Empyema thoracis refers to accumulation of pus in the pleural space. The pathogenic organisms isolated in cases of empyema depend on prior antibiotic use and route of infection i.e. whether infection arises as a complication of pneumonia or following oesophageal surgery. It also depends on the age of the patient and presence of co-morbid illness.
\end{abstract}

\section{MATERIALS AND METHODS}

It is a descriptive study. It was designed to investigate the bacterial isolates of thoracic empyema and to find out the antibiogram pattern of the isolated organisms in a tertiary care hospital over one-year period. In this study, bacteriological spectrum was analysed in 50 empyema cases. Samples of pleural fluid were sent for bacterial culture (Aerobic) and for Gram's stain as well as for cytologic studies and mycobacterial and fungal smears and cultures, also if clinically indicated Cartridge Based Nucleic Acid Amplification test (CBNAAT). Patients with tubercular empyema were excluded.

\section{RESULTS}

24 cases were culture positive. Among the 24 culture positive cases, Streptococcus pneumoniae was the commonest bacterium isolated followed by Staphylococcus aureus. A strong inverse correlation was found between prior antibiotic use with chance of culture positivity. In present study Gram-positive organisms were found to be most sensitive to Vancomycin, Linezolid and some are sensitive to Clindamycin, Ciprofloxacin, Levofloxacin and Co-amoxyclav. Gram negative organisms were mostly sensitive to Ceftriaxone, Cefoperazone, Cefepime, Piperacillin-Tazobactam, Meropenem and Colistin. All the strains of Pseudomonas aeruginosa were sensitive to Amikacin. Resistance to Co-amoxyclav was noted in many strains of the Gram negative organisms except Klebsiella.

\section{CONCLUSION}

Gram positive organisms were the commonest organisms isolated in our study. This study also supports the view that prior antibiotic use reduces the chances of detecting the micro-organism through culture and determination of sensitivity.

\section{KEY WORDS}

Empyema, Pneumonia, Bacteria, Culture.

HOW TO CITE THIS ARTICLE: Dwari AK, Jha S, Sarkar S, et al. A study of bacterial isolates and their sensitivity pattern to antibiotics in empyema thoracis cases in a tertiary care hospital. J. Evolution Med. Dent. Sci. 2018;7(38):4178-4181, D0I: $10.14260 /$ jemds/2018/934

\section{BACKGROUND}

Empyema thoracis is the collection of pus in the pleural space. Empyema commonly results from pneumonia, bloodborne infection, thoracic surgery, trauma, abdominal infection or neoplasm.[1] Pneumonia causes more than half the cases of empyema, post-surgical infection accounts for additional 20 percent.[2] Richard W Light ${ }^{[2]}$ defined the term empyema as those pleural effusions with thick, purulent appearing fluid.

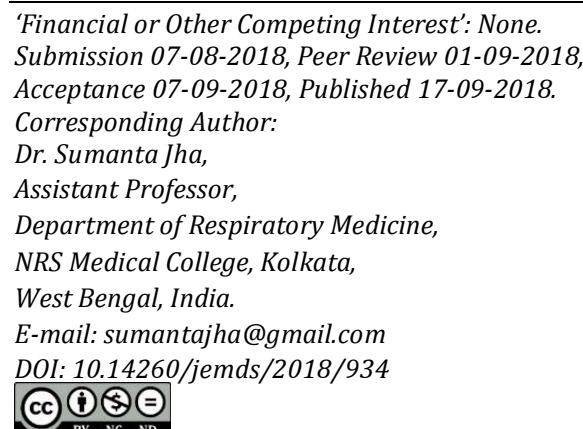


There are approximately one million patients hospitalised in the United States each year with pneumonia. Of those hospitalised, 20 to $40 \%$ have a parapneumonic effusion. The mortality is higher in patients with pneumonia who have a pleural effusion. In one study, the mortality risk was 6.5 times higher if the effusions were bilateral, whereas the mortality risk was 3.7 times higher if the effusion was unilateral. Although, some of the increased mortality is due to comorbid conditions, some of it is due to mismanagement of the pleural effusion. In assessing whether patients with communityacquired pneumonia require hospitalisation, the presence of a pleural effusion is given the same weight as a $\mathrm{PaO}_{2}$ level of less than $60 \mathrm{mmHg}$.

Symptoms of parapneumonic effusion and empyema may be acute or chronic. Anaerobic pulmonary infections frequently have an associated pleural effusion and are characterised by a more chronic course. Weight loss and anaemia are common with anaerobic infections. In patients with pneumonia, the clinical picture such as the degree of leukocytosis or the incidence of chest pain is very similar whether or not they have a parapneumonic effusion. Similarly, if the patient has a parapneumonic effusion the clinical picture is similar whether or not the effusion is complicated.

- The possibility of a parapneumonic effusion should be considered during the initial evaluation of every patient with a bacterial pneumonia.

- At this evaluation, it is important to determine whether a complicated parapneumonic effusion is present, because a delay in instituting proper pleural drainage in such patients substantially increases morbidity.

- The possibility of a parapneumonic effusion should also be suspected in patients who do not respond to antimicrobial therapy.

- Radiologically presence of pleural effusion is diagnosed with the help of chest $\mathrm{x}$-ray PA view, lateral view, lateral decubitus view, thoracic ultrasonography, computed tomography etc. We quantify pleural effusion by measuring the distance between the inside of the chest wall and the bottom of the lung on either the decubitus radiograph or the CT scan of the chest. This distance is also measured with ultrasound.

\section{MATERIALS AND METHODS}

It is a descriptive study. The pleural fluid was examined grossly for colour, turbidity and odour. Aliquots were sent for determination of the pleural fluid differential and total WBC counts, glucose, protein, ADA and LDH levels. Samples of pleural fluid were also sent for bacterial culture (Aerobic) and for Gram's stain as well as for cytologic studies and mycobacterial and fungal smears and cultures, also if clinically indicated Cartridge Based Nucleic Acid Amplification test (CBNAAT). Patients with tubercular empyema were excluded.

Pleural fluid samples from fifty suspected cases of empyema were analysed. After collection and assessing the pleural sample it was cultured on blood agar, chocolate agar or MacConkey's agar plates.

These inoculated plates were then incubated for a period of 24 hours, after which they were examined for evidence of bacterial growth. In case of bacterial growth on the medium, the bacteria were further identified using standard tests.
The following Tests were Performed according to Standard Methods

Gram's staining, Hanging drop test, Catalase test, Oxidase test, Indole test, Methyl red test, Citrate utilisation test, Urease test, Hydrogen sulfide production test, Sugar fermentation test, Nitrate reduction test, Coagulase test (for Staphylococci), Bile solubility test (For Streptococcus pneumoniae), Bacitracin test (for $\beta$-haemolytic Streptococci) and optochin sensitivity test (for Pneumococci).

Antibiotic sensitivity test of the isolates were performed on Mueller-Hinton agar plates by the disc diffusion method of Kirby-Bauer. After the plates were dried, broth suspension of the organisms was made and adjusted to McFarland's opacity factor 0.5. A lawn culture was made over the surface of the media using a sterile swab, then appropriate antibiotics discs were placed and incubated at $37^{\circ} \mathrm{C}$ for 24 hours after which readings were taken. The zone of inhibition was measured and reported. Any resistance colony found within the inhibiting zone gave an indication as to the presence of resistance mutants. Sensitivity was performed using control strains of Klebsiella pneumoniae ATCC 700603, Staphylococcus aureus ATCC 25923, E. coli ATCC 25922 and Pseudomonas ATCC 27853.

The concentrations of the antibiotics employed in the antibiotic disc were as per CLSI guidelines.[7] Antibiogram was read, that is zones of inhibition were measured and sensitivities to various antibiotics were determined using standard guidelines for each antibiotic regarding the zone of inhibition and sensitivity.

\section{Statistical Analysis}

The data collected were analysed statistically using IBM SPSS Statistics software version 20. Chi-square test was used as the test of significance. A p-value of $<0.05$ was considered significant.

\section{RESULTS}

In culture of pleural fluid of all the 50 patients with empyema twenty six (52\%) were culture negative, eight (16\%) showed growth of Streptococcus pneumoniae, seven (14\%) were positive for Staphylococcus aureus, one (2\%) for Escherichia coli, two $(4 \%)$ for Haemophilus influenzae, three $(6 \%)$ for Klebsiella sp. and the rest three $(6 \%)$ for Pseudomonas aeruginosa.

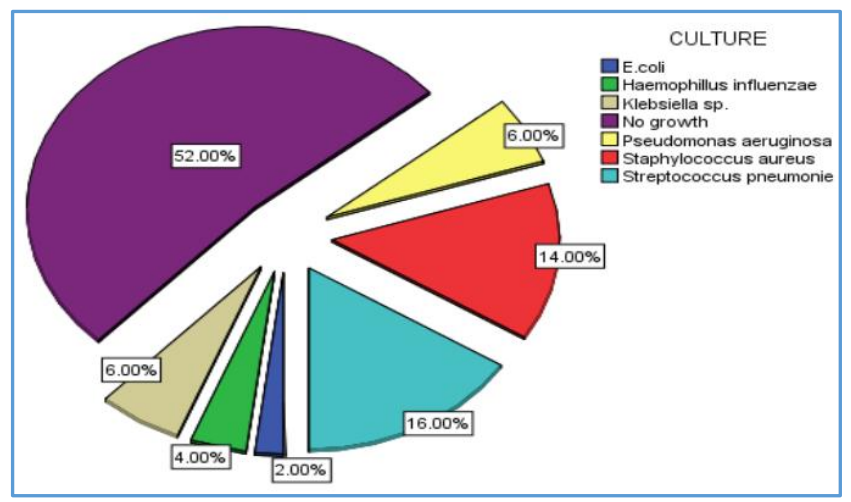

Figure 1. Bacterial Isolates in Empyema Thoracis Cases

Among the total twenty-four culture positive samples eight (33.33\%) were positive for Streptococcus pneumoniae, seven $(29.17 \%)$ for Staphylococcus aureus, one $(4.16 \%)$ for 
Escherichia coli, two (8.33\%) for Haemophilus influenzae, three $(12.50 \%)$ for Klebsiella sp. and three (12.50\%) for Pseudomonas aeruginosa.

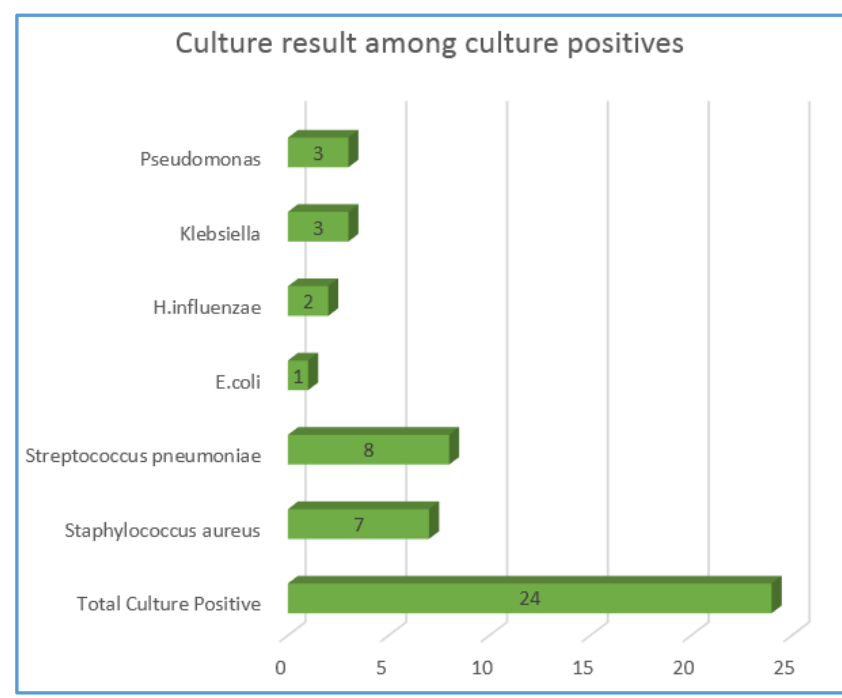

Figure 2. Bacterial Strains in Culture Positive Samples

Staphylococcus aureus was the commonest organism isolated in age group of 20 - 39 years and Streptococcus pneumoniae was the commonest in age groups of $40-60$ years and $>60$ years. However, there was no statistically significant relationship between age of the patient and bacterial strains isolated.

Streptococcus pneumoniae was the commonest causative organism of empyema in diabetic patients and patients without any comorbid condition. However, there was no statistically significant relationship between different comorbid conditions and causative organisms.

Staphylococcus aureus was the commonest organism isolated in patients with empyema caused by iatrogenic factors and Streptococcus pneumoniae in parapneumonic empyema. However, there was no statistically significant correlation found between aetiology and bacterial strains isolated in culture.

No statistically significant correlation was found between prior antibiotic use and bacterial strain isolated in culture of pleural fluid. However, there was a statistically significant inverse correlation between prior antibiotic use and chance of culture positivity of pleural fluid. Among twenty one patients without use of prior antibiotics, nineteen (90\%) gave positive result in pleural fluid culture and out of twenty nine patients who used prior antibiotics only five $(17 \%)$ were culture positive.

\begin{tabular}{|c|c|c|c|}
\hline \multirow{3}{*}{$\begin{array}{l}\text { Prior } \\
\text { Antibiotic Use }\end{array}$} & \multicolumn{2}{|c|}{ Culture } & \multirow{5}{*}{$P$ value -.00} \\
\hline & Positive & Negative & \\
\hline & $\begin{array}{c}\text { No. of } \\
\text { Patients }\end{array}$ & $\begin{array}{c}\text { No. of } \\
\text { Patients }\end{array}$ & \\
\hline No & 19 & 2 & \\
\hline Yes & 5 & 24 & \\
\hline \multicolumn{4}{|c|}{$\begin{array}{c}\text { Table 1. Correlation between Prior Antibiotic Use and } \\
\text { Culture Positivity }\end{array}$} \\
\hline
\end{tabular}

All the Staphylococcus aureus strains isolated were sensitive to Vancomycin (100\%) followed by Linezolid (86\%) and Clindamycin (43\%). Only $14 \%$ were sensitive to Ciprofloxacin, Levofloxacin, Amikacin and Co-amoxyclav.

Streptococcus pneumoniae strains were mostly sensitive to Vancomycin (88\%) followed by Linezolid (75\%), Cefoperazone (75\%), Meropenem (75\%) and PiperacillinTazobactam (75\%). They were also sensitive to Co-amoxyclav (63\%), Ceftriaxone (63\%), Ciprofloxacin (50\%), Clindamycin (50\%) and Levofloxacin (37\%).

\begin{tabular}{|c|c|c|c|c|c|}
\hline \multicolumn{2}{|l|}{ Drugs } & \multicolumn{2}{|c|}{$\begin{array}{c}\text { Staphylococcus } \\
\text { aureus }\end{array}$} & \multicolumn{2}{|c|}{$\begin{array}{c}\text { Streptococcus } \\
\text { pneumoniae }\end{array}$} \\
\hline & & \multirow{2}{*}{$\begin{array}{c}\mathbf{n}=\mathbf{7} \\
1\end{array}$} & \multirow{2}{*}{$\begin{array}{c}\% \\
14 \% \\
\end{array}$} & \multirow{2}{*}{$\begin{array}{c}\mathbf{n}=\mathbf{8} \\
5\end{array}$} & \multirow{2}{*}{$\begin{array}{c}\% \\
63 \% \\
\end{array}$} \\
\hline Co-amoyyclay & $\mathrm{S}$ & & & & \\
\hline Co-amoxyclav & $\mathrm{R}$ & 6 & $86 \%$ & 3 & $37 \%$ \\
\hline \multirow{2}{*}{ Ceftriaxone } & $\mathrm{S}$ & 0 & $0 \%$ & 5 & $63 \%$ \\
\hline & $\mathrm{R}$ & 7 & $100 \%$ & 3 & $37 \%$ \\
\hline \multirow{2}{*}{ Cefoperazone } & $\mathrm{S}$ & 0 & $0 \%$ & 6 & $75 \%$ \\
\hline & $\mathrm{R}$ & 7 & $100 \%$ & 2 & $25 \%$ \\
\hline \multirow{2}{*}{ Ciprofloxacin } & $\mathrm{S}$ & 1 & $14 \%$ & 4 & $50 \%$ \\
\hline & $\mathrm{R}$ & 6 & $86 \%$ & 4 & $50 \%$ \\
\hline \multirow{2}{*}{ Meropenem } & $\mathrm{S}$ & 0 & $0 \%$ & 6 & $75 \%$ \\
\hline & $\mathrm{R}$ & 7 & $100 \%$ & 2 & $25 \%$ \\
\hline \multirow{2}{*}{ Linezolid } & $\mathrm{S}$ & 6 & $86 \%$ & 6 & $75 \%$ \\
\hline & $\mathrm{R}$ & 1 & $14 \%$ & 2 & $25 \%$ \\
\hline \multirow{2}{*}{$\begin{array}{l}\text { Piperacillin+ } \\
\text { Tazobactam }\end{array}$} & $\mathrm{S}$ & 0 & $0 \%$ & 6 & $75 \%$ \\
\hline & $\mathrm{R}$ & 7 & $100 \%$ & 2 & $25 \%$ \\
\hline \multirow{2}{*}{ Clindamycin } & $\mathrm{S}$ & 3 & $43 \%$ & 4 & $50 \%$ \\
\hline & $\mathrm{R}$ & 4 & $57 \%$ & 4 & $50 \%$ \\
\hline \multirow{2}{*}{ Amikacin } & $\mathrm{S}$ & 1 & $14 \%$ & 1 & $13 \%$ \\
\hline & $\mathrm{R}$ & 6 & $86 \%$ & 7 & $87 \%$ \\
\hline \multirow{2}{*}{ Levofloxacin } & $\mathrm{S}$ & 1 & $14 \%$ & 3 & $37 \%$ \\
\hline & $\mathrm{R}$ & 6 & $86 \%$ & 5 & $63 \%$ \\
\hline \multirow{2}{*}{ Vancomycin } & $\mathrm{S}$ & 7 & $100 \%$ & 7 & $86 \%$ \\
\hline & $\mathrm{R}$ & 0 & $0 \%$ & 1 & $14 \%$ \\
\hline \multirow{2}{*}{ Gentamicin } & $\mathrm{S}$ & 1 & $14 \%$ & 0 & $0 \%$ \\
\hline & $\mathrm{R}$ & 6 & $86 \%$ & 8 & $100 \%$ \\
\hline
\end{tabular}

Table 2. Sensitivity result of Staphylococcus aureus and Streptococcus pneumoniae to different Antibiotics

The only Escherichia coli strain isolated was sensitive to Ceftriaxone, Cefoperazone, Ciprofloxacin, Meropenem, Amikacin, Colistin, Levofloxacin and Gentamicin. It was resistant to Netilmicin and Piperacillin-Tazobactam.

Among the two strains of Haemophilus influenzae the two $(100 \%)$ were sensitive to Ceftriaxone, Cefoperazone, Ciprofloxacin, Piperacillin-Tazobactam and Levofloxacin. One (50\%) was sensitive to Netilmicin, Meropenem, Amikacin, Colistin and Doxycycline. Both (100\%) were resistant to Coamoxyclav.

Among the three strains of Klebsiella sp. All three (100\%) were sensitive to Ceftriaxone, Meropenem and Levofloxacin. Two (67\%) were sensitive to Co-amoxyclav, Ciprofloxacin, Netilmicin, Colistin and Gentamicin. One (33\%) was sensitive to Cefoperazone, Piperacillin-Tazobactam and Amikacin.

Out of the three Pseudomonas aeruginosa strains all three (100\%) were sensitive to Meropenem, Colistin, Amikacin and Cefepime. Two (67\%) were sensitive to Ciprofloxacin, Ceftazidime, Piperacillin-Tazobactam, Levofloxacin and Gentamicin. All three (100\%) were resistant to Co-amoxyclav, Netilmicin and Doxycycline. 


\begin{tabular}{|c|c|c|c|c|c|c|c|c|c|}
\hline & \multicolumn{2}{|c|}{$\begin{array}{l}\overline{8} \\
\overline{0} \\
\end{array}$} & \multicolumn{2}{|r|}{ 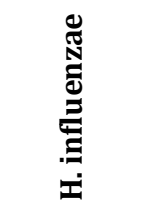 } & \multicolumn{2}{|c|}{ 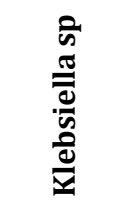 } & \multicolumn{2}{|c|}{ 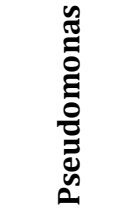 } \\
\hline & & II & $\delta^{0}$ & $\stackrel{N}{=}$ & $\delta^{0}$ & $\stackrel{n}{I I}$ & $\delta^{\circ}$ & $\stackrel{\mathbb{I}}{=}$ & $d^{0}$ \\
\hline \multirow{2}{*}{$\begin{array}{c}\text { Co- } \\
\text { amoxyclav }\end{array}$} & $\mathrm{S}$ & 0 & $0 \%$ & 0 & $0 \%$ & 2 & $67 \%$ & 0 & $0 \%$ \\
\hline & $\mathrm{R}$ & 1 & $100 \%$ & 2 & $100 \%$ & 1 & $33 \%$ & 3 & $100 \%$ \\
\hline \multirow{2}{*}{ Ceftriaxone } & $\mathrm{S}$ & 1 & $100 \%$ & 2 & $100 \%$ & 3 & $100 \%$ & 0 & $0 \%$ \\
\hline & $\mathrm{R}$ & 0 & $0 \%$ & 0 & $0 \%$ & 0 & $0 \%$ & 0 & $0 \%$ \\
\hline \multirow{2}{*}{ Ceftazidime } & $S$ & 0 & $0 \%$ & 0 & $0 \%$ & 0 & $0 \%$ & 2 & $67 \%$ \\
\hline & $\mathrm{R}$ & 1 & $100 \%$ & 0 & $0 \%$ & 0 & $0 \%$ & 1 & $33 \%$ \\
\hline \multirow{2}{*}{ Cefoperazone } & $S$ & 1 & $100 \%$ & 2 & $100 \%$ & 1 & $33 \%$ & 0 & $0 \%$ \\
\hline & $\mathrm{R}$ & 0 & $0 \%$ & 0 & $0 \%$ & 2 & $67 \%$ & 1 & $33 \%$ \\
\hline \multirow{2}{*}{ Ciprofloxacin } & $\mathrm{S}$ & 1 & $100 \%$ & 2 & $100 \%$ & 2 & $67 \%$ & 2 & $67 \%$ \\
\hline & $\mathrm{R}$ & 0 & $0 \%$ & 0 & $0 \%$ & 1 & $33 \%$ & 1 & $33 \%$ \\
\hline \multirow{2}{*}{ Netilmicin } & $\mathrm{S}$ & 0 & \begin{tabular}{|l|}
$0 \%$ \\
\end{tabular} & 1 & $50 \%$ & 2 & $67 \%$ & 0 & $0 \%$ \\
\hline & $\mathrm{R}$ & 1 & $100 \%$ & 1 & $50 \%$ & 1 & $33 \%$ & 3 & $100 \%$ \\
\hline \multirow{2}{*}{ Meropenem } & $\mathrm{S}$ & 1 & $100 \%$ & 1 & $50 \%$ & 3 & $100 \%$ & 3 & $100 \%$ \\
\hline & $\mathrm{R}$ & 0 & $0 \%$ & 1 & $50 \%$ & 0 & $0 \%$ & 0 & $0 \%$ \\
\hline \multirow{2}{*}{$\begin{array}{c}\text { Piperacillin }+ \\
\text { Tazobactam } \\
\end{array}$} & $S$ & 0 & $0 \%$ & 2 & $100 \%$ & 1 & $33 \%$ & 2 & $67 \%$ \\
\hline & $\mathrm{R}$ & 1 & $100 \%$ & 0 & $0 \%$ & 2 & $67 \%$ & 1 & $33 \%$ \\
\hline \multirow{2}{*}{ Amikacin } & $\mathrm{S}$ & 1 & $100 \%$ & 1 & $50 \%$ & 1 & $33 \%$ & 3 & $100 \%$ \\
\hline & $\mathrm{R}$ & 0 & $0 \%$ & 1 & $50 \%$ & 2 & $67 \%$ & 0 & $0 \%$ \\
\hline \multirow{2}{*}{ Colistin } & $S$ & 1 & $100 \%$ & 1 & $50 \%$ & 2 & $67 \%$ & 3 & $100 \%$ \\
\hline & $\mathrm{R}$ & 0 & $0 \%$ & 0 & $0 \%$ & 0 & $0 \%$ & 0 & $0 \%$ \\
\hline \multirow{2}{*}{ Levofloxacin } & $\mathrm{S}$ & 1 & $100 \%$ & 2 & $100 \%$ & 3 & $100 \%$ & 2 & $67 \%$ \\
\hline & $\mathrm{R}$ & 0 & $0 \%$ & 0 & $0 \%$ & 0 & $0 \%$ & 1 & $33 \%$ \\
\hline \multirow{2}{*}{ Cefepime } & $\mathrm{S}$ & 0 & $0 \%$ & 0 & $0 \%$ & 0 & $0 \%$ & 3 & $100 \%$ \\
\hline & $\mathrm{R}$ & 1 & $100 \%$ & 0 & $0 \%$ & 0 & $0 \%$ & 0 & $0 \%$ \\
\hline \multirow{2}{*}{ Gentamicin } & $\mathrm{S}$ & 1 & $100 \%$ & 0 & $0 \%$ & 2 & $67 \%$ & 2 & $67 \%$ \\
\hline & $\mathrm{R}$ & 0 & $0 \%$ & 0 & $0 \%$ & 1 & $33 \%$ & 1 & $33 \%$ \\
\hline \multirow{2}{*}{ Doxycycline } & $\mathrm{S}$ & 0 & $0 \%$ & 1 & $50 \%$ & 0 & $0 \%$ & 0 & $0 \%$ \\
\hline & $\mathrm{R}$ & 1 & $100 \%$ & 1 & $50 \%$ & 1 & $33 \%$ & 3 & $100 \%$ \\
\hline
\end{tabular}

Table 3. Sensitivity result of E. coli, $\mathrm{H}$. influenzae, Klebsiella sp. and Pseudomonas aeruginosa to different Antibiotics

\section{DISCUSSION}

In the present study bacteriological spectrum was analysed in 50 empyema cases, of which 24 were culture positive. It was observed that empyema is higher in males (68\%) than in females (32\%) with the ratio of 2: 1 and the peak was in the age group of 40 to 60 years. In a study conducted by Alfageme et al[8] showed average age of patient with empyema was 54, which is similar to our present study.

Among the 24 culture positive cases Streptococcus pneumoniae was the commonest bacteria isolated in 8 cases followed by Staphylococcus aureus isolated in 7 cases, Klebsiella sp. in 3 cases, Pseudomonas aeruginosa in 3 cases, Haemophilus influenzae in 2 cases and Escherichia coli in 1 case. It was also observed that out of 24 cases, all were single bacterial isolates. In this study, the prevalence of Gram positive isolates was $63 \%$ as compared to $37 \%$ of Gram negative. In an analysis of thirty-seven cases of pleural empyema done by Meyerovitch et al[9] revealed that Streptococcus pneumoniae was the most frequently isolated pathogen (41\%) followed by Staphylococcus aureus (14\%). Our present study show similar pattern of aetiological agents.
In our present study empyema was caused by iatrogenic factors in $8(16 \%)$ cases, as a consequence of lung abscess in 2 (4\%) cases and the rest 40 (80\%) cases were parapneumonic.

In this study no statistically significant correlation was found between bacterial strain isolated in culture with age of the patient, co-morbidities, aetiology of empyema and prior antibiotic use. However, a strong inverse correlation was found between prior antibiotic use with chance of culture positivity.

In the present study Gram positive organisms were found to be most sensitive to Vancomycin, Linezolid and some were sensitive to Clindamycin, Ciprofloxacin, Levofloxacin and Coamoxyclav. Some strains of Streptococcus pneumoniae were well sensitive to Cefoperazone, Ceftriaxone, PiperacillinTazobactam and Meropenem.

Gram negative organisms were mostly sensitive to Ceftriaxone, Cefoperazone, Cefepime, PiperacillinTazobactam, Meropenem and Colistin. All the strains of Pseudomonas aeruginosa were sensitive to Amikacin. Resistance to Co-amoxyclav was noted in many strains of all the Gram negative organisms except Klebsiella.

\section{CONCLUSION}

Gram positive organisms were the commonest organisms isolated in our study. This study also supports the view that prior antibiotic use reduces the chances of detecting the micro-organism through culture and determination of sensitivity.

\section{REFERENCES}

[1] Fishman AP, Elias JA, Fishman JA, et al. Fishman's pulmonary diseases and disorders. The McGraw-Hill Companies, Inc., 2008: p. 2144-7.

[2] Light RW. Pleural diseases. Chapter - 12. $5^{\text {th }}$ edn. Philadelphia, PA: Lipincott Williams \& Wilkins 2007.

[3] Seaton A, Seaton D, Lt. Leitch GA. Crofton and Douglas's respiratory diseases. Blackwell Science Ltd., 2000: p. 454-5.

[4] Jerng JS, Hsueh PR, Teng LJ, et al. Empyema thoracis and lung abscess caused by viridans streptococci. Am J Respir Crit Care Med 1997;156(5):1508-14.

[5] Tsang KY, Leung WS, Chan VL, et al. Complicated parapneumonic effusion and empyema thoracis: microbiology and predictors of adverse outcomes. Hong Kong Med J 2007;13(3):178-86.

[6] Barlett JG, Gorbach SL, Thadepalli H, et al. Bacteriology of empyema. Lancet 1974;303(7853):338-40.

[7] Clinical and Laboratory Standards Institute (CLSI). Analysis and presentation of cumulative antimicrobial susceptibility test data. 3rd edn. Approved guideline M39-A3. Wayne PA. CLSI, 2009.

[8] Alfageme I, Munoz F, Pena N, et al. Empyema of the thorax in adults. Etiology, microbiologic findings and management. Chest 1993;103(3):839-43.

[9] Brims FJ, Lansley SM, Waterer GW, et al. Empyema thoracis: new insights into an old disease. European Respiratory Review 2010;19(117):220-8. 